\title{
Quantum liquids of particles with generalized statistics
}

\author{
Serguei B. Isakov \\ Department of Physics, University of Oslo, P.O. Box 1048 Blindern, N-0316 Oslo, Norway \\ and Division de Physique Théorique, Institut de Physique Nucléaire, Orsay Fr-91406, France*
}

\begin{abstract}
We propose a phenomenological approach to quantum liquids of particles obeying generalized statistics of a fermionic type, in the spirit of the Landau Fermi liquid theory. The approach is developed for fractional exclusion statistics. We discuss both equilibrium (specific heat, compressibility, and Pauli spin susceptibility) and nonequilibrium (current and thermal conductivities, thermopower) properties. Low temperature quantities have the same temperature dependences as for the Fermi liquid, with the coefficients depending on the statistics parameter. The novel quantum liquids provide explicit realization of systems with a non-Fermi liquid Lorentz ratio in two and more dimensions. Consistency of the theory is verified by deriving the compressibility and $f$-sum rules.
\end{abstract}

Keywords: generalized quantum statistics, exclusion statistics, quantum liquids PACS: 05.30.-d, 71.10.Pm

*Present address. isakov@ipno.in2p3.fr 


\section{INTRODUCTION}

There has been increasing interest in generalized statistics for identical particles due to possible applications to fractional quantum Hall effect and high- $T_{c}$ superconductivity [1.22]. Fractional exclusion statistics [3], for which a single-state distribution function can be defined in the thermodynamic limit [4] [ has appeared in a system of anyons in the lowest Landau level [7], in Heisenberg [8] and Schrödinger [9] quantization of identical particles (as fractional statistics in one dimension), in Calogero-Sutherland models [10] and received much attention because of its relevance to the fractional quantum Hall effect, both to the bulk [11] and edge [12 physics.

It has been realized that the idea of a generalized exclusion principle [3] is flexible enough to be able to accommodate some other statistical distributions [13], for instance those for Gentile parafermions [14]. All these distributions belong to a class - we refer to the relevant statistics as local - for which the average number of particles in a given quantum state $i$ depends only on the Gibbs factor $x_{i}=e^{\left(\mu-\varepsilon_{i}\right) / k_{B} T}$ for the same quantum state, $n_{i}=n\left(x_{i}\right)$. The function $n\left(x_{i}\right)$ is considered to be quite arbitrary up to natural physical constraints such as the existence of the Boltzmann limit for any statistical distribution for an ideal quantum gas when the mean occupation numbers are small, $n_{i} \rightarrow x_{i}$ as $x_{i} \rightarrow 0$ [15]. If one specifies the equation for the distribution function to be

$$
\frac{n_{i}}{\mathcal{F}\left(n_{i}\right)}=e^{\left(\mu-\varepsilon_{i}\right) / k_{B} T}
$$

then the Boltzmann limit condition implies $\mathcal{F}(n) \rightarrow 1$ as $n \rightarrow 0$.

Low temperature thermodynamic quantities were calculated for ideal gases of particles obeying exclusion and other local statistics [16 18]. To discuss possibilities to detect generalized statistics in experiments, one normally has to have a theory of interacting particles. In this paper we suggest a phenomenological way to treat interaction in systems of particles obeying local statistics, in the spirit of Landau's approach to the Fermi liquid [19] (see also [20]). We restrict our attention to statistics of a fermionic type, whose zero temperature distribution functions are step functions (with the height of the step generally different from unity).

In Sect. [1] we formulate the notion of quantum liquids of particles obeying generalized statistics and discuss low temperature equilibrium properties for spinless particles. Explicit formulas are worked out for exclusion statistics. In Sect. III we study non-equilibrium quantities in a simpler case of noninteracting exclusion statistics particles in the relaxation time approximation. We show that the Wiedemann-Franz law holds, with a non-Fermi Lorentz ratio depending on the statistics parameter, and discuss relation to the calculation of the Lorentz ratio for Luttinger liquids [21]. In Sect. एV] we introduce the transport (kinetic) equation for the liquid and show that the Lorentz ratio is not modified by the interaction. To verify the theory, we check in Sect. $\square$ that the sum rules for the density response function 
are respected. In Sect. V1 we discuss how spin of the particles can be included. We conclude in Sect. VII with remarks on generic local statistics, non-linear dispersion of quasiparticles and possible applications to compressible quantum Hall states.

\section{LIQUID OF SPINLESS PARTICLES}

For an ideal gas of particles obeying fermionic local statistics, by definition, the zero temperature distribution function is constant (not necessarily equal to unity) for $p<p_{0}$ (inside a pseudo-Fermi sphere) and vanishes for $p>p_{0}$. In generalizing Landau's approach [19] to such statistics, we assume that when interaction between particles is adiabatically switched on, there exists a liquid state which can be described, at low temperatures, in terms of elementary excitations, quasiparticles ("dressed" particles), which inherit quantum statistical properties of particles of the ideal gas.

The latter means that the entropy of the liquid, in terms of the quasiparticle distribution function $n_{\boldsymbol{p}}$ (we consider spinless particles), is given by the same combinatorial expression as for a corresponding gas. For exclusion statistics this implies [4, [5]

$$
\begin{aligned}
S= & \sum_{\boldsymbol{p}}\left\{\left[1+(1-g) n_{\boldsymbol{p}}\right] \ln \left[1+(1-g) n_{\boldsymbol{p}}\right]\right. \\
& \left.-\left(1-g n_{\boldsymbol{p}}\right) \ln \left(1-g n_{\boldsymbol{p}}\right)-n_{\boldsymbol{p}} \ln n_{\boldsymbol{p}}\right\} .
\end{aligned}
$$

The statistics parameter $g$ is assumed to be positive to avoid the Bose limit; $g=1$ corresponds to Fermi statistics.

The energy of the system is a functional of the distribution of quasiparticles, $E=E\left[n_{\boldsymbol{p}}\right]$. Variation of the energy

$$
\delta E=\sum_{\boldsymbol{p}} \varepsilon_{\boldsymbol{p}} \delta n_{\boldsymbol{p}}+\sum_{\boldsymbol{p} \boldsymbol{p}^{\prime}} f_{\boldsymbol{p} \boldsymbol{p}^{\prime}} \delta n_{\boldsymbol{p}} \delta n_{\boldsymbol{p}^{\prime}}
$$

defines the energy of a quasiparticle $\varepsilon_{\boldsymbol{p}}$ (which is a functional of the distribution function, $\left.\varepsilon_{\boldsymbol{p}}=\varepsilon_{\boldsymbol{p}}\left[n_{\boldsymbol{p}}\right]\right)$ as well as the quasiparticle interaction functional $f_{\boldsymbol{p} \boldsymbol{p}^{\prime}}=f_{\boldsymbol{p} \boldsymbol{p}^{\prime}}\left[n_{\boldsymbol{p}}\right]$.

Varying the entropy at constant total energy and constant particle number, with the use of (3), yields an equation for the distribution function

$$
\frac{n_{\boldsymbol{p}}}{\left(1-g n_{\boldsymbol{p}}\right)^{g}\left[1+(1-g) n_{\boldsymbol{p}}\right]^{1-g}}=e^{\left(\mu-\varepsilon_{\boldsymbol{p}}\left[n_{\boldsymbol{p}}\right]\right) / k_{B} T} .
$$

At zero temperature the distribution function is the same as for an ideal gas,

$$
n_{\boldsymbol{p}}=\left\{\begin{array}{cc}
1 / g & |\boldsymbol{p}|<p_{0} \\
0 & |\boldsymbol{p}|>p_{0}
\end{array}\right.
$$

This can be justified with the use of Eq. (4) and the zero temperature dispersion of quasiparticles (8). 
The number of quasiparticles is equal to the number of particles and hence the relation between the pseudo Fermi momentum and the density is the same as for an ideal gas,

$$
N=\int_{|\boldsymbol{p}|<p_{0}} \frac{d^{d} \boldsymbol{p} V}{(2 \pi \hbar)^{d}} \frac{1}{g} .
$$

With $d^{d} \boldsymbol{p}=p^{d-1} d p d \Omega_{\boldsymbol{p}}$, where $d \Omega_{\boldsymbol{p}}$ stands for integration over angles of $\boldsymbol{p}$ so that $\int d \Omega_{\boldsymbol{p}}=$ $S_{d-1}$, and $S_{d}=2 \pi^{d / 2} / \Gamma\left(\frac{1}{2} d\right)$ is the surface of a $d$-dimensional sphere of unit radius $\left(S_{0} \equiv 2\right)$, Eq. (6) implies a relation between the pseudo Fermi momentum and the particle number density $\rho=N / V$

$$
\left(\frac{p_{0}}{2 \pi \hbar}\right)^{d}=g \frac{\rho d}{S_{d-1}}
$$

Note that in microscopic theory, Eq. (অ) has be proved, as a generalization of the Luttinger theorem [22].

For $T=0$ the quasiparticle energy functional becomes a function since the distribution function is fixed. We assume the standard linear dispersion for quasiparticles near the pseudo Fermi energy

$$
\varepsilon_{\boldsymbol{p}}=\mu+v_{0}\left(p-p_{0}\right)+\ldots
$$

where $v_{0} \equiv p_{0} / m^{*}$, and $m^{*}$ is an effective mass. The quasiparticle interaction functional also becomes a function. We will only need to know the quasiparticle interaction function on the pseudo Fermi surface, $f_{\boldsymbol{p}_{0}} \boldsymbol{p}_{0}^{\prime}$. For a spherically symmetric case, it depends only on the angle $\theta$ between $\boldsymbol{p}$ and $\boldsymbol{p}^{\prime}: f_{\boldsymbol{p}_{0} \boldsymbol{p}_{0}^{\prime}}=f(\theta)$, with the function $f(\theta)$ even, due to the symmetry $f_{\boldsymbol{p} \boldsymbol{p}^{\prime}}=f_{\boldsymbol{p}^{\prime} \boldsymbol{p}}$.

A relation between the effective mass and the quasiparticle interaction function follows from the Galilean invariance. The latter implies $\sum_{\boldsymbol{p}} \boldsymbol{p} n_{\boldsymbol{p}}=\sum_{\boldsymbol{p}} m\left(\partial \varepsilon_{\boldsymbol{p}}\left[n_{\boldsymbol{p}}\right] / \partial \boldsymbol{p}\right) n_{\boldsymbol{p}}$ or, upon varying w.r.t. $n_{\boldsymbol{p}}$ 19]

$$
\frac{\boldsymbol{p}}{m}=\frac{\partial \varepsilon_{\boldsymbol{p}}}{\partial \boldsymbol{p}}-\sum_{\boldsymbol{p}^{\prime}} f\left(\boldsymbol{p}, \boldsymbol{p}^{\prime}\right) \frac{\partial n_{\boldsymbol{p}^{\prime}}}{\partial \boldsymbol{p}^{\prime}} .
$$

Multiplying (9) by $\boldsymbol{p}$, choosing $\boldsymbol{p}$ to belong to the pseudo Fermi surface and using the zero temperature distribution function with $\partial n_{\boldsymbol{p}^{\prime}} / \partial \boldsymbol{p}^{\prime}=-\left(\boldsymbol{p}^{\prime} / p^{\prime}\right) \frac{1}{g} \delta\left(p^{\prime}-p_{0}\right)$, we obtain

$$
\frac{m^{*}}{m}=1+\frac{1}{g} \nu\left(\varepsilon_{0}\right) \overline{f(\theta) \cos \theta} .
$$

The bar denotes the average over angles in spherical coordinates in a $d$-dimensional $\boldsymbol{p}^{\prime}$-space, with the polar axis along $\boldsymbol{p}$ and polar angle $\theta, \overline{A(\theta)} \equiv \int A(\theta)\left(d \Omega_{\boldsymbol{p}^{\prime}} / S_{d-1}\right)$. We have also introduced the density of states on the pseudo Fermi surface

$$
\nu\left(\varepsilon_{0}\right)=\frac{S_{d-1}}{(2 \pi \hbar)^{d}} m^{*} p_{0}^{d-2} V .
$$


One can introduce a dimensionless quasiparticle interaction function $F(\theta)=\nu\left(\varepsilon_{0}\right) f(\theta)$, and define Landau parameters $F_{0}$ and $F_{1}$ by

$$
\overline{F(\theta)}=F_{0}, \quad \overline{F(\theta) \cos \theta}=\frac{1}{d} F_{1} .
$$

In one dimension, where the polar angle takes two values, $\theta=0, \pi, F_{0}$ and $F_{1}$ are given by $F_{0}=\frac{1}{2}[F(0)+F(\pi)]$ and $F_{1}=\frac{1}{2}[F(0)-F(\pi)]$. In two dimensions they are coefficients of an expansion $F(\theta)=\sum_{l=0}^{\infty} F_{l} \cos (l \theta)$, and in three dimensions they correspond to the standard expansion in Legendre polynomials, $F(\theta)=\sum_{l=0}^{\infty} F_{l} P_{l}(\cos \theta)$.

In the above notation, (10) reads

$$
\frac{m^{*}}{m}=1+\frac{1}{g} \frac{F_{1}}{d}
$$

For calculating the specific heat one can use the dispersion law (\$) for zero temperature. Finite temperature corrections to the dispersion (from the second term in the r.h.s. of (3)) give terms of higher order in the temperature and can be neglected. The specific heat is thus obtained by the change $m \rightarrow m^{*}$ in the expression for an ideal gas given in Ref. [17],

$$
C_{V}=\frac{\pi^{2}}{3} \nu\left(\varepsilon_{0}\right) k_{B}^{2} T
$$

To calculate the compressibility $\kappa$ at zero temperature, defined as $1 / \kappa=N \rho(\partial \mu / \partial N)_{V}$, we note that in varying $N$ there are two sources for changing the pseudo-Fermi energy, $\left.\varepsilon_{\boldsymbol{p}}\left[\left.n_{\boldsymbol{p}}\right|_{T=0}\right]\right|_{p=p_{0}}$, due to the changes of the distribution of quasiparticles and the pseudoFermi momentum. In this way we obtain $1 / \kappa=N \rho\left(g+F_{0}\right) / \nu\left(\varepsilon_{0}\right)$, or in terms of the first sound velocity,

$$
v_{s}^{2}=\frac{p_{0}^{2}}{d m m^{*}}\left(1+\frac{F_{0}}{g}\right)
$$

$\left(v_{s}=p_{0} / m \sqrt{d}\right.$ in the limit of vanishing interaction).

\section{TRANSPORT PROPERTIES IN THE RELAXATION TIME APPROXIMATION}

Four transport coefficients relate electrical and thermal currents to changes in electrochemical potential and the temperature (we consider an isotropic liquid) [23] :

$$
\begin{aligned}
\boldsymbol{j} & =\mathcal{L}^{11}\left(\boldsymbol{E}-\frac{\nabla \mu}{e}\right)+\mathcal{L}^{12}(-\nabla T), \\
\boldsymbol{j}^{Q} & =\mathcal{L}^{21}\left(\boldsymbol{E}-\frac{\nabla \mu}{e}\right)+\mathcal{L}^{22}(-\nabla T) .
\end{aligned}
$$

We start with discussion of a simpler case of the transport equation for noninteracting exclusion statistics particles in the relaxation time approximation 


$$
\frac{\partial n}{\partial t}+\boldsymbol{v}(\varepsilon) \frac{\partial n}{\partial \boldsymbol{r}}+e \boldsymbol{E} \frac{\partial n}{\partial \boldsymbol{p}}=-\frac{n-n^{0}}{\tau(\varepsilon)}
$$

allowing for dependence of the relaxation time $\tau(\varepsilon)$ on the energy. Here $n^{0}$ is an equilibrium (uniform) distribution. We do not specify the dispersion law of particles, only assuming that it is isotropic, $\varepsilon_{\boldsymbol{p}}=\varepsilon(p) ; \boldsymbol{v}(\varepsilon)=\partial \varepsilon / \partial \boldsymbol{p}$ is the particle velocity. The carrier charge $e$ may be different from the charge of an electron and may have either sign.

Consider the distribution function with slowly varying chemical potential and temperature $n=n\left(e^{\left[\mu(\boldsymbol{r})-\varepsilon_{\boldsymbol{p}}\right] / k_{B} T(\boldsymbol{r})}\right)$. Then for the stationary solution to first order in gradients of the electrochemical potential and temperature we obtain from (17)

$$
n=n^{0}-\tau(\varepsilon)\left\{\boldsymbol{v} \cdot(e \boldsymbol{E}-\nabla \mu)+\frac{\mu-\varepsilon}{T}(\boldsymbol{v} \cdot \nabla T)\right\} \frac{\partial n^{0}}{\partial \varepsilon} .
$$

With this distribution function the transport coefficients in (16) $\operatorname{read} \mathcal{L}^{11}=\mathcal{L}_{0}, \mathcal{L}^{12}=$ $-\frac{1}{T} \mathcal{L}^{21}=\frac{1}{e T} \mathcal{L}_{1}$, and $\mathcal{L}^{22}=\frac{1}{e^{2} T} \mathcal{L}_{2}$, where

$$
\mathcal{L}_{r}=-\int_{0}^{\infty} d \varepsilon(\varepsilon-\mu)^{r} \sigma_{F}(\varepsilon) \frac{\partial n^{0}}{\partial \varepsilon}
$$

and

$$
\begin{aligned}
\sigma_{F}(\varepsilon) & =e^{2} \tau(\varepsilon) \sum_{\boldsymbol{p}} \delta\left(\varepsilon-\varepsilon_{\boldsymbol{p}}\right) v^{2}(\varepsilon) \\
& =e^{2} \tau(\varepsilon) \nu(\varepsilon) v^{2}(\varepsilon)
\end{aligned}
$$

$(\nu(\varepsilon)$ is the density of states) is the energy-dependent conductivity for a Fermi gas with the Fermi energy $\varepsilon_{0}$. The symmetry between $\mathcal{L}^{12}$ and $\mathcal{L}^{21}$ manifests Onsager's principle.

At low temperatures integrals of the form (18), involving the distribution function for exclusion statistics, can be evaluated using the expansion [17]

$$
\begin{aligned}
\int_{0}^{\infty} d \varepsilon n_{g}(\varepsilon) \mathcal{G}(\varepsilon)= & \frac{1}{g} \int_{0}^{\mu} d \varepsilon \mathcal{G}(\varepsilon) \\
& +\frac{\pi^{2}}{6} \mathcal{G}^{\prime}(\mu)\left(k_{B} T\right)^{2}+\cdots
\end{aligned}
$$

valid for functions $\mathcal{G}(\varepsilon)$ which vary on the scale $\varepsilon_{0}$. In this way we obtain the leading terms of the transport coefficients

$$
\begin{aligned}
& \mathcal{L}^{11}=\frac{1}{g} \sigma_{F}\left(\varepsilon_{0}\right), \quad \mathcal{L}^{22}=\frac{\pi^{2} k_{B}^{2} T}{3 e^{2}} \sigma_{F}\left(\varepsilon_{0}\right), \\
& \mathcal{L}^{12}=-\frac{1}{T} \mathcal{L}^{12}=-\left.\frac{\pi^{2} k_{B}^{2} T}{3 e}\left(\frac{\partial \sigma_{F}(\varepsilon)}{\partial \varepsilon}\right)\right|_{\varepsilon=\varepsilon_{0}} .
\end{aligned}
$$

The low temperature conductivity $\sigma=\mathcal{L}^{11}=\frac{1}{g} \sigma_{F}\left(\varepsilon_{0}\right)$ is independent of the temperature. The thermal conductivity relates the thermal current to the temperature gradient $\boldsymbol{j}^{Q}=$ 
$-K \nabla T$ provided $\boldsymbol{j}=0$, thus yielding $K=\mathcal{L}^{22}-\mathcal{L}^{12} \mathcal{L}^{21} / \mathcal{L}^{11}$. From (21) we observe that the ratio of the thermal conductivity to the electrical conductivity is proportional to the temperature thus respecting the Wiedemann-Franz law but with the Lorentz number

$$
L \equiv \frac{K}{\sigma T}=g \frac{\pi^{2}}{3}\left(\frac{k_{B}}{e}\right)^{2}=g L_{F},
$$

differed by the factor $g$ from its value $L_{F}$ for a Fermi liquid.

This observation tells us that the difference of the Lorentz number from its value for the Fermi liquid may signal a kinematic effect (change of statistics).

The thermopower $Q=\mathcal{L}^{12} / \mathcal{L}^{11}$ is

$$
Q=-\left.g \frac{\pi^{2} k_{B}^{2} T}{3 e}\left(\frac{\partial \ln \sigma_{F}(\varepsilon)}{\partial \varepsilon}\right)\right|_{\varepsilon=\varepsilon_{0}} .
$$

For a free gas, assuming that $\tau(\varepsilon) \propto \varepsilon^{\lambda}$, one obtains $\sigma_{F}(\varepsilon) \propto \varepsilon^{\lambda+D / 2}$ and (23) reduces to $Q=-g(\lambda+d / 2)\left(\pi^{2} k_{B} / 3 e\right)\left(k_{B} T / \varepsilon_{0}\right)$.

Relation to a Luttinger liquid. - A Luttinger liquid [24] is characterized by the velocities of charge and current excitations, $v_{N}$ and $v_{J}$, and the sound velocity $v_{s}$, related by $v_{s}^{2}=v_{N} v_{J}$. As two independent parameters, one can choose $v_{s}$ and a parameter $\mathcal{K}$ such that $v_{J}=\mathcal{K} v_{s}$ and $v_{N}=v_{s} / \mathcal{K}$.

By comparison of the low temperature specific heat for a (spinless) Luttinger liquid $C / V=\pi^{2} k_{B} T / 3 \hbar v_{s}$ [24] with that of an ideal gas of exclusion statistics particles, (14) for $F_{0}=F_{1}=0$, we observe that the thermodynamic quantities for the two systems are identical if

$$
g=1 / \mathcal{K}
$$

(see also an argument by bosonization [25).

Our calculation of the Lorentz ratio show that the the correspondence between a Luttinger liquid and an ideal gas of exclusion statistics particles can be extended to nonequilibrium properties. Namely, by comparison (22) with the result for the Lorentz ratio for a Luttinger liquid $L=L_{F} / \mathcal{K}$ [21], we see that the two expressions coincide under the above identification (24).

\section{TRANSPORT EQUATION FOR THE LIQUID}

Now we turn to the liquid. Consider distributions corresponding to small departures from the equilibrium quasiparticle distribution function, $n_{\boldsymbol{p}}(\boldsymbol{r}, t)=n_{\boldsymbol{p}}^{0}\left(\varepsilon_{\boldsymbol{p}}^{0}\right)+\delta n_{\boldsymbol{p}}(\boldsymbol{r}, t)$, where the superscript 0 labels equilibrium quantities. For short range interactions the corresponding change in energy of a quasiparticle can be written in a local form (i.e. depending only on 
$\delta n_{\boldsymbol{p}}(\boldsymbol{r}, t)$ at the same point): $\delta \varepsilon_{\boldsymbol{p}}(\boldsymbol{r}, t)=\sum_{\boldsymbol{p}^{\prime}} f_{\boldsymbol{p}^{\prime}} \delta n_{\boldsymbol{p}^{\prime}}(\boldsymbol{r}, t)$ [2]. If $n^{0}$ is expressed in terms of the actual energy of quasiparticles, $\varepsilon=\varepsilon^{0}+\delta \varepsilon$, the perturbed distribution function reads

$$
n_{\boldsymbol{p}}(\boldsymbol{r}, t)=n_{\boldsymbol{p}}^{0}\left(\varepsilon_{\boldsymbol{p}}\right)+\delta \tilde{n}_{\boldsymbol{p}}(\boldsymbol{r}, t),
$$

where $\delta \tilde{n}=\delta n-\delta \varepsilon\left(\partial n^{0} / \partial \varepsilon\right)$ is the quantity (rather than $\left.\delta n\right)$ determining the charge and thermal currents, $\boldsymbol{j}=-e \sum_{\boldsymbol{p}} \boldsymbol{v} \delta \tilde{n}$ and $\boldsymbol{j}^{Q}=-e \sum_{\boldsymbol{p}}(\varepsilon-\mu) \boldsymbol{v} \delta \tilde{n}$. The transport equation

$$
\frac{\partial \delta n}{\partial t}+\frac{\partial \varepsilon^{0}}{\partial \boldsymbol{p}} \frac{\partial \delta n}{\partial \boldsymbol{r}}-\frac{\partial \delta \varepsilon}{\partial \boldsymbol{r}} \frac{\partial n^{0}}{\partial \boldsymbol{p}}=I(n)
$$

has the same form as for the Fermi liquid, except for the collision integral $I(n)$, where factors $(1-n)$ accounting for the Pauli exclusion principle should be modified.

The form of the collision integral for ideal gases with arbitrary local statistics for identical particles was discussed by the author [15]. It was argued in Ref. [15] that in constructing collision integrals one should replace factors accounting for the Pauli exclusion principle as follows:

$$
(1-n) \rightarrow \mathcal{F}(n),
$$

where the function $\mathcal{F}(n)$ arises in the equation for the equilibrium distribution function (11)

For fractional exclusion statistics the function $\mathcal{F}(n)$ can be read off from Eq. (四): $\mathcal{F}(n)=$ $(1-g n)^{g}[1+(1-g) n]^{1-g}$. The latter form was also found in discussions of the Boltzmann equation for exclusion statistics [26].

Using the change (27) we can write down the collision integral for the case of the residual interaction due to an elastic scattering by impurities:

$$
I\left(n_{\boldsymbol{p}}\right)=\frac{2 \pi}{\hbar} \sum_{\boldsymbol{p}^{\prime}} w_{\boldsymbol{p}^{\prime}} \delta\left(\varepsilon_{\boldsymbol{p}}-\varepsilon_{\boldsymbol{p}^{\prime}}\right)\left[n_{\boldsymbol{p}} \mathcal{F}\left(n_{\boldsymbol{p}^{\prime}}\right)-n_{\boldsymbol{p}^{\prime}} \mathcal{F}\left(n_{\boldsymbol{p}}\right)\right] .
$$

Here $\frac{2 \pi}{\hbar} w_{\boldsymbol{p} \boldsymbol{p}^{\prime}} \delta\left(\varepsilon_{\boldsymbol{p}}-\varepsilon_{\boldsymbol{p}^{\prime}}\right)$ is the transition probability for the scattering of a quasiparticle from state $\boldsymbol{p}$ to state $\boldsymbol{p}^{\prime}$. We assumed that the impurity atoms are randomly distributed and form a dilute gas so that they scatter independently; the detailed balancing principle $w_{\boldsymbol{p}^{\prime}}=w_{\boldsymbol{p}^{\prime} \boldsymbol{p}}$ was also used.

Using (25) we linearize the collision integral to obtain

$$
I(\delta \tilde{n})=\frac{2 \pi}{\hbar} \sum_{\boldsymbol{p}^{\prime}} w_{\boldsymbol{p}^{\prime}} \delta\left(\varepsilon_{\boldsymbol{p}}-\varepsilon_{\boldsymbol{p}^{\prime}}\right)\left[\delta \tilde{n}_{\boldsymbol{p}}-\delta \tilde{n}_{\boldsymbol{p}^{\prime}}\right] .
$$

The transport coefficients can be calculated using the linearized transport equation which to first order in gradients of the temperature and chemical potential reads

$$
\left(e \boldsymbol{E}+\frac{\mu-\varepsilon}{T} \boldsymbol{\nabla} T\right) \cdot \boldsymbol{v} \frac{\partial n^{0}}{\partial \varepsilon}=I(\delta \tilde{n}),
$$


where $\boldsymbol{v}=\partial \varepsilon^{0} / \partial \boldsymbol{p}$.

For an elastic scattering (in particular, for collision integrals of the form (28) when $w_{\boldsymbol{p p}^{\prime}}$ depends only on the angle between $\boldsymbol{p}$ and $\boldsymbol{p}^{\prime}$ ), collisions move particles on the constant energy surface, and one can seek the solution of the transport equation in the form (cf. relevant discussion for a Fermi liquid [27]) $\delta \tilde{n}=-\left(\partial n^{0} / \partial \varepsilon\right)[e \boldsymbol{E}+(\mu-\varepsilon)(\boldsymbol{\nabla} T / T)] \cdot \boldsymbol{l}(\boldsymbol{p})$, where $\boldsymbol{l}(\boldsymbol{p})$ satisfies the equation $I(\boldsymbol{l})=-\boldsymbol{v}$. Note that the Landau parameters do not enter explicitly (they are absorbed in $\delta \tilde{n}$ ). As a result, we obtain the same form of the transport coefficients $\mathcal{L}^{a b}$ as in the previous section for an ideal gas, with the energy dependent conductivity (19) replaced by $\sigma_{F}(\varepsilon)=e^{2} \sum_{\boldsymbol{p}} \delta\left(\varepsilon-\varepsilon_{\boldsymbol{p}}\right) \boldsymbol{l} \cdot \boldsymbol{v}$.

Then calculating the low temperature expansion as in Sect. III yields the result (21), with the above $\sigma_{F}(\varepsilon)$, and leads to the previously found Lorentz ratio (22).

The fact that the interaction does not change the Lorentz number (for a residual conductivity due to scattering by impurities) suggests that this number may be a good quantity to check in searching for generalized statistics in experiments.

\section{LINEAR RESPONSE AND SUM RULES}

To derive linear response functions from the transport equation, a harmonic perturbation of the density by a scalar potential $\varphi(\boldsymbol{q}, \omega)$ is applied corresponding to an external force $\boldsymbol{F}=-i \boldsymbol{q} \varphi(\boldsymbol{q}, \omega) e^{i(\boldsymbol{q} r-\omega t)}+$ c. c. acting on a liquid [20]. This modifies the transport equation (26), written for the Fourier components, to

$$
\begin{aligned}
& (\omega-\boldsymbol{q} \boldsymbol{v}) \delta n_{\boldsymbol{p}}(\boldsymbol{q}, \omega)+\boldsymbol{q} \boldsymbol{v} \frac{\partial n^{0}}{\partial \varepsilon_{\boldsymbol{p}}} \sum_{\boldsymbol{p}^{\prime}} f_{\boldsymbol{p \boldsymbol { p } ^ { \prime }}} \delta n_{\boldsymbol{p}^{\prime}}(\boldsymbol{q}, \omega) \\
& +\boldsymbol{q} \boldsymbol{v} \varphi(\boldsymbol{q}, \omega) \frac{\partial n^{0}}{\partial \varepsilon_{\boldsymbol{p}}}=i I\left(\delta n_{\boldsymbol{p}}(\boldsymbol{q}, \omega)\right) .
\end{aligned}
$$

By solving this equation for small $\varphi(\boldsymbol{q}, \omega)$, one can find the density response function $\chi(\boldsymbol{q}, \omega)=\langle\rho(\boldsymbol{q}, \omega)\rangle / \varphi(\boldsymbol{q}, \omega)$, where $\langle\rho(\boldsymbol{q}, \omega)\rangle=\sum_{\boldsymbol{p}} \delta n_{\boldsymbol{p}}(\boldsymbol{q}, \omega)$ is the (average) induced density fluctuation.

Consider the collisionless regime where one can neglect the collision integral. In the static limit $\omega \ll q v_{0}$ we find from (30) $\delta n_{\boldsymbol{p}}(\boldsymbol{q}, 0)=\varphi(\boldsymbol{q}, 0)\left(\partial n^{0} / \partial \varepsilon_{\boldsymbol{p}}\right) /\left(1+F_{0} / g\right)$. This leads to the response function

$$
\chi(\boldsymbol{q}, 0)=-\frac{\nu\left(\varepsilon_{0}\right)}{g+F_{0}} .
$$

By comparison with the expression for the first sound velocity (15), we see that Eq. (31) is consistent with the compressibility sum rule $\lim _{\boldsymbol{q} \rightarrow 0} \chi(\boldsymbol{q}, 0)=-N / m v_{s}^{2}$ [20].

We note that the transport equation for the liquid is itself valid only for perturbations $\delta n$ varying on a macroscopic scale implying that eq. (30) holds only for $\boldsymbol{q}$ small enough. 
In the quasihomogeneous limit, $q v_{0} \ll \omega$, in calculating $\delta n_{\boldsymbol{p}}(\boldsymbol{q}, \omega)$ in (30) one has to keep terms up to second order in $q v_{0} / \omega$, resulting in $\chi(\boldsymbol{q}, \omega)=N q^{2} / m \omega^{2}$, which manifests the $f$-sum rule as $\omega \rightarrow \infty$ [20].

In the hydrodynamic regime, where $\omega$ is much less than the collision frequency and $q \ell \ll 1$, with $\ell$ the mean free path, collisions dominate. In this case, all the deviations from the equilibrium distributions are damped out by the collisions, except for those of the form (near the pseudo-Fermi surface) $\delta n^{(0)}$ (constant) and $\delta n^{(1)} \cos \varphi$, where $\varphi$ is the angle between $\boldsymbol{k}$ and $\boldsymbol{p}$. The latter contribute into the particle number and momentum fluxes respectively and for them the collision integral vanishes due to the particle number and momentum conservation.

Integrating Eq. (30) over momenta, with $\boldsymbol{q}$ as the polar axis, with the weights 1 and $\boldsymbol{p}$ respectively, one obtains two coupled equations for $\delta n^{(0)}$ and $\delta n^{(1)}$ solution of which results in $\chi(\boldsymbol{q}, \omega)=(\mathrm{N} / \mathrm{m}) /\left(\omega^{2} / q^{2}-v_{s}^{2}\right)$ leading to the same static and homogeneous limits as in the collisionless case. We thus conclude that our phenomenological theory respects the compressibility and $f$-sum rules both in the collisionless and in the hydrodynamic regimes.

\section{LIQUID OF SPINNING PARTICLES. SPIN SUSCEPTIBILITY}

Consider spinning particles, of spin $1 / 2$ for definiteness, with $\alpha=\frac{1}{2},-\frac{1}{2}$ labeling the spin projections (we also use $\alpha=\uparrow, \downarrow$ in subscripts).

There is an ambiguity with assigning statistics to spinning particles. In general, one can introduce a statistics matrix $g_{\alpha \alpha^{\prime}}$ (four parameters) determining how particles with different spin projections affect each other. There are two special cases, $g_{\alpha \alpha^{\prime}}=g \delta_{\alpha \alpha^{\prime}}$ and $g_{\alpha \alpha^{\prime}}=g$ involving a single statistics parameter. In this paper we discuss the case $g_{\alpha \alpha^{\prime}}=g \delta_{\alpha \alpha^{\prime}}$ which means that particles of different spin projections are not connected statistically (like ordinary fermions which are recovered for $g=1$ ).

Now the energy of a quasiparticle and the Landau interaction function acquire additional spin indices. All the formulas of the previous sections are valid upon replacing the quasiparticle interaction function by its symmetric combination $f(\theta) \rightarrow f_{s}(\theta)=\frac{1}{2}\left[f_{\uparrow \uparrow}(\theta)+f_{\uparrow \downarrow}(\theta)\right]$ (and similarly for the associated Landau parameters) provided, in addition, $\nu\left(\varepsilon_{0}\right)$ is as twice as given by (11), due to the two spin polarizations. The latter definition of $\nu\left(\varepsilon_{0}\right)$ is assumed in the rest of this section.

In the presence of the magnetic field, the variation of the energy of a quasiparticle reads (assuming the gyromagnetic ratio two)

$$
\delta \varepsilon_{\boldsymbol{p} \alpha}=-2 \mu_{B} \alpha B+\sum_{\boldsymbol{p}^{\prime} \alpha^{\prime}} f_{\boldsymbol{p} \alpha, \boldsymbol{p}^{\prime} \alpha^{\prime}} \delta n_{\boldsymbol{p}^{\prime} \alpha^{\prime}} .
$$

With $\delta n_{\boldsymbol{p} \alpha}=\left(\partial n_{\boldsymbol{p} \alpha} / \partial \varepsilon_{\boldsymbol{p} \alpha}\right) \delta \varepsilon_{\boldsymbol{p} \alpha}$, the solution of (32) to first order in $B$ at zero temperature yields modification of the magnetic energy due to the interaction $\delta \varepsilon_{\boldsymbol{p} \alpha}=$ 
$-2 \mu_{B} \alpha B /\left(1+F_{0}^{a} / g\right)$, where $F_{0}^{a}=\frac{1}{2}\left(F_{\uparrow \uparrow, 0}-F_{\uparrow \downarrow, 0}\right)$ is an antisymmetric combination of the Landau parameters. From the variation of the magnetization $\delta M=\sum_{p \alpha} 2 \mu_{B} \alpha \delta n_{p \alpha}$ we find the magnetic susceptibility

$$
\chi=\frac{\mu_{B}^{2}}{V} \frac{\nu\left(\varepsilon_{0}\right)}{g+F_{0}^{a}} .
$$

Without including liquid effects $\left(F_{0}^{a}=0\right)$, this expression reduces to the magnetic susceptibility of an ideal gas of exclusion statistics particles [18] (which means that the authors of Ref. [18] used implicitly the statistics matrix of the form $\left.g_{\alpha \alpha^{\prime}}=g \delta_{\alpha \alpha^{\prime}}\right)$. Note that the Wilson ratio $R_{W}=\left(\pi^{2} k_{B}^{2} T \chi V / 3 \mu_{B}^{2} C\right)$, which is equal to $R_{W}=1 /\left(g+F_{0}^{a}\right)$ according to (14) and (33), differs from its Fermi gas value (unity) due to both the statistics of particles and the interaction.

\section{CONCLUDING REMARKS}

We have developed a phenomenological approach to a quantum liquid of particles obeying fractional exclusion statistics. For generic local statistics of a fermionic type, determined by (1), the starting basic equation for the distribution function of quasiparticles (国) should be replaced by

$$
\frac{n_{\boldsymbol{p}}}{\mathcal{F}\left(n_{\boldsymbol{p}}\right)}=e^{\left(\mu-\varepsilon_{\boldsymbol{p}}\left[n_{\boldsymbol{p}}\right]\right) / k_{B} T} .
$$

We note that in fact all the formulas for the zero temperature quantities in Sections $\mathbb{M}$ and VI as well as the formulas for the linear response (Sect. V) are valid in this more general case provided $g$ is understood not as the exclusion statistics parameter but just as the height of the step determining the distribution function of quasiparticles at zero temperature (5).

The form of the transport equation (26) also holds for generic local statistics. We stress that the Lorentz ratio - when it is determined by the scattering by impurities - only contains information about the statistics of quasiparticles thus being a good tool for search for new statistics. In contrast, the Wilson number encodes information both on interaction and statistics and cannot be interpreted in that simple way.

We have discussed the usual, linear dispersion of quasiparticles near the pseudo-Fermi surface. In fact, one can consider more complicated dispersion laws. We comment in this respect on a possible application of ideas of generalized quantum liquids to models of twodimensional fermions coupled to a gauge field that were used to describe metallic non-Fermi liquid states.

Specifically, we consider a system of fermions coupled to an abelian Chern-Simons field and interacting with a two-body potential $v(\boldsymbol{r}) \propto V_{0} / r^{\eta}$ (or in Fourier components, $V(\boldsymbol{q})=$ $\left.V_{0} / q^{2-\eta}\right)$, with $1 \leq \eta \leq 2(\eta=1$ corresponds to the Coulomb interaction). This model 
is relevant to compressible quantum Hall states with filling factor $\nu=\frac{1}{2}$ 28 as well as to high- $T_{c}$ superconductivity [29].

In an attempt to fit the result of the random phase approximation for the above model into the quasiparticle picture in the framework of the Landau Fermi liquid theory, a renormalized energy of quasiparticles near the Fermi surface has been introduced, of the form 28

$$
\varepsilon_{\boldsymbol{p}}-\mu \propto\left|p-p_{F}\right|^{\frac{1+\eta}{2}} \operatorname{sgn}\left(p-p_{F}\right) .
$$

The associated specific heat has the form $C=\gamma T^{\frac{2}{1+\eta}}$. However, the coefficient $\gamma$ differs from the result obtained in another way, from the free energy of the gauge field (the latter method, being gauge invariant, is regarded to be exact) [30]. The reason for such inconsistency is in that the Landau quasiparticles in this system are ill-defined since the imaginary part of the self-energy of quasiparticles is of order of their real part (except for $\eta=1$, where the damping of quasiparticles is logarithmically small compared to their energy, in which case the two ways to calculate the specific heat agree).

For the quantum liquids discussed in the present paper, if quasiparticles have the dispersion (35) (with $p_{F} \rightarrow p_{0}$ ), the temperature dependence of the specific heat will be the same as for a Fermi liquid, with the coefficient $\gamma$ depending on the statistics of quasiparticles. Based on this, we point out a principal possibility to reconcile the results of calculations of the specific heat in the above system in the two ways, by assuming that quasiparticles (for $\eta \neq 1$ ) form a non-Fermi quantum liquid, corresponding to some generalized statistics. In more general terms, our conjecture is that fluctuations of the gauge fields may lead to a kinematic effect (changing statistics of excitations from Fermi to some other) for metallic states. More detailed discussion of this issue is left for a future publication.

\section{Acknowledgments}

I would like to thank D.P. Arovas for stimulating discussions and comments. Centre for Advanced Study (SHS) in Oslo is acknowledged for warm hospitality and support during the 1995/1996 program "Quantum phenomena in low-dimensional systems" where this work was started. I also thank J.M. Leinaas and A.P. Polychronakos for insightful discussions following my lectures on the Landau Fermi liquid theory at SHS. The work was partly supported by the Norwegian Research Council. 


\section{REFERENCES}

[1] F. Wilczek (Ed.), Fractional Statistics and Anyon Superconductivity (World Scientific, 1990).

[2] The Quantum Hall Effect, Ed. by R.E. Prange and S.M. Girvin (Second Edition, Springer, 1990).

[3] F.D.M. Haldane, Phys. Rev. Lett. 67 (1991) 937.

[4] S.B. Isakov, Mod. Phys. Lett. B 8 (1994) 319.

[5] Y.S. Wu, Phys. Rev. Lett. 73 (1994) 922.

[6] A.K. Rajakopal, Phys. Rev. Lett. 74 (1995) 1048.

[7] A. Dasnières de Veigy and S. Ouvry, Phys. Rev. Lett. 72 (1994) 600.

[8] J.M. Leinaas and J. Myrheim, Phys. Rev. B 37 (1988) 9286; Int. J. Mod. Phys. B 5 (1991) 2573.

[9] A. Polychronakos, Nucl. Phys. B 324 (1989) 597.

[10] S.B. Isakov, Int. J. Mod. Phys. A 9 (1994) 2563; M.V.N. Murthy and R. Shankar, Phys. Rev. Lett. 73 (1994) 3331; Z.N.C. Ha, Phys. Rev. Lett. 73 (1994) 1574.

[11] M.D. Johnson and G.S. Canright, Phys. Rev. B 49 (1994) 2947; D. Li and S. Ouvry, Nucl. Phys. B 430 (1994) 563; W.P. Su, Y.S. Wu, and J. Yang, Phys. Rev. Lett. 77 (1996) 3423; S.B. Isakov, G.S. Canright, and M.D. Johnson, Phys. Rev. B 55 (1997) 6727; K. Tevosyan and A.H. MacDonald, Phys. Rev. B 56 (1997) 7517.

[12] T. Fukui and N. Kawakami, Phys. Rev. B 51 (1995) 5239; S.B. Isakov and S. Viefers, Int. J. Mod. Phys. A 12 (1997) 1895; R.A.J. van Elburg, K. Schoutens, Quasi-particles in fractional quantum Hall effect edge theories, cond-mat/9801272.

[13] A.P. Polychronakos, Phys. Lett. B 365 (1996) 202; A.V. Ilinskaia, K.N. Ilinski, and J.M.F. Gunn, Nucl. Phys. B 458 (1996) 562; G. Kaniadakis, A. Lavagno, and P. Quarati, Nucl. Phys. B 466 (1996) 527; K. Schoutens, Phys. Rev. Lett. 79 (1997) 2608.

[14] G. Gentile, Nuovo Cimento 17 (1940) 493.

[15] S.B. Isakov, Int. J. Theor. Phys. 32 (1993) 737.

[16] D. Sen and R.K. Bhaduri, Phys. Rev. Lett. 74 (1995) 3912; C. Nayak and F. Wilczek, Phys. Rev. Lett. 73 (1994) 2740.

[17] S.B. Isakov, D.P. Arovas, J. Myrheim, and A.P. Polychronakos, Phys. Lett. A 212 (1996) 299.

[18] A. Carbone, G. Kaniadakis, and P. Quarati, Physica A 238 (1997) 361.

[19] L.D. Landau, JETP 3 (1956) 920; 5 (1957) 101.

[20] D. Pines, P. Nozières. The theory of quantum liquids. Vol. 1. Normal Fermi liquids (W.A. Benjamin, 1966).

[21] C.L. Kane and M.P.A. Fisher, Phys. Rev. Lett 76 (1996) 3192.

[22] J.M. Luttinger, Phys. Rev. 118 (1960) 1153. 
[23] See, e.g. N.W. Ashcroft and N.D. Mermin, Solid State Physics (Saunders College Publishing, 1976)

[24] For recent reviews, see H.J. Schulz, Fermi liquids and non-Fermi liquids, condmat/9503150; J. Voit, Rep. Prog. Phys. 58 (1995) 977.

[25] Y.S. Wu and Y. Yu, Phys. Rev. Lett. 75 (1995) 890.

[26] R.K. Bhaduri, R.S. Bhalerao, and M.V.N. Murthy, J. Stat. Phys. 82 (1996) 1659.

[27] E.M. Lifshitz and L.P. Pitaevskii, Physical Kinetics (Pergamon, 1981). Chap. IX.

[28] B. I. Halperin, P. A. Lee, and N. Read, Phys. Rev. B 47, 7312 (1993).

[29] For relevant references, see e.g. Y.B. Kim, A. Furusaki, X.G. Wen, and P.A. Lee, Phys. Rev. B 50 (1994) 17917.

[30] Y.B. Kim and P.A. Lee, Phys. Rev. B 54 (1996) 2715. 